\title{
Pectus excavatum
}

\section{Background}

Pectus excavatum, also known as funnel chest, is a condition whereby an individual's breastbone, or sternum, grows inwards. This results in a sunken appearance of the chest wall. Pectus excavatum is often apparent from early infancy and may worsen significantly during adolescence. Affected individuals may experience physical symptoms, such as shortness of breath and chest pain, as well as psychological distress. In moderate to severe cases, surgery provides a successful means of correcting the deformity, alleviating symptoms and improving the quality of life for those affected by the condition.

\section{Risk factors}

The underlying cause of pectus excavatum is not well understood; however, it is thought to relate to irregular growth of the cartilage that connects the sternum to the ribs. It is more common in males than females, as well as those with a family history of the condition. While the majority of cases are not associated with any other condition, pectus excavatum may occur in conjunction with a number of inherited musculoskeletal disorders, such as Marfan syndrome and congenital scoliosis.

\section{Signs and symptoms}

In addition to the characteristic indentation of the chest wall, pectus excavatum may cause symptoms by compressing the underlying heart and lungs. These include shortness of breath, chest pain, palpitations, fatigue and reduced exercise tolerance. Regardless of severity, the cosmetic deformity associated with any case of pectus excavatum may also result in psychological issues relating to body image and selfesteem.

\section{Investigations}

While pectus excavatum is usually evident on examination of the chest, a number of tests may be carried out to determine the impact of the condition on the heart and lungs. These include imaging, such as a chest X-ray and computed tomography (CT) chest scan, which demonstrate the extent to which the heart and lungs are compressed. An echocardiogram, which is an ultrasound imaging study of the heart, is helpful in characterizing how well the heart is functioning. Other tests that may be conducted include an electrocardiogram, which illustrates whether an abnormal heart rhythm has developed, and lung function tests, which measure breathing parameters.

\section{Management}

Mild cases of pectus excavatum may be managed with physiotherapy alone, which involves exercises aimed at improving posture and chest expansion. In addition, psychological counseling should be provided to those who have difficulty coping with the condition. For moderate to severe cases, surgical correction is indicated. The most common surgical operation performed, known as the Nuss procedure, is a minimally invasive operation that aims to elevate the sunken sternum. This procedure is performed under the guidance of a thin camera and involves making small incisions on either side of the chest, insertion of a curved steel bar under the sternum, and fixation of the bar to the ribs on both sides of the chest. The metal bar is surgically removed after several years when the deformity has been corrected. Other more invasive operative approaches involve cutting out segments of the ribs to accommodate one or more metal bars under the sternum. Surgical correction of pectus excavatum most often results in significant improvements in symptoms and quality of life. These operations carry a number of risks, however, including infection, bleeding and pain postoperatively, though these risks may be lower for the Nuss procedure. As always, the risks should be weighed against the benefits and discussed with your surgeon.

For more information, please visit the following websites: http://www.mayoclinic.org/diseases-conditions/pectusexcavatum/basics/definition/con-20028599 http://my.clevelandclinic.org/services/heart/disorders/hic_ pectus_excavatum

http://radiopaedia.org/articles/pectus-excavatum

doi: 10.21037/acs.2016.08.07

Section Writer: Christopher Harris

Illustration Editor: Beth Croce

Section Editor: Christopher Cao
For specific information concerning your medical condition, ACS suggests that you consult your physician. This page may be photocopied non-commercially by physicians to share with patients. 